\title{
HIGH-RESOLUTION OBSERVATIONS OF THE POLAR MAGNETIC FIELDS OF THE SUN
}

\author{
H. LIN, J. VARSIK, and H. ZIRIN \\ Big Bear Solar Observatory, California Institute of Technology, Pasadena, CA 91125, U.S.A.
}

(Received 8 December, 1993; in revised form 10 August, 1994)

\begin{abstract}
High-resolution magnetograms of the solar polar region were used for the study of the polar magnetic field. In contrast to low-resolution magnetograph observations which measure the polar magnetic field averaged over a large area, we focused our efforts on the properties of the small magnetic elements in the polar region. Evolution of the filling factor - the ratio of the area occupied by the magnetic elements to the total area - of these magnetic elements, as well as the average magnetic field strength, were studied during the maximum and declining phase of solar cycle 22, from early 1991 to mid-1993.

We found that during the sunspot maximum period, the polar regions were occupied by about equal numbers of positive and negative magnetic elements, with equal average field strength. As the solar cycle progresses toward sunspot minimum, the magnetic field elements in the polar region become predominantly of one polarity. The average magnetic field of the dominant polarity elements also increases with the filling factor. In the meanwhile, both the filling factor and the average field strength of the non-dominant polarity elements decrease. The combined effects of the changing filling factors and average field strength produce the observed evolution of the integrated polar flux over the solar cycle.

We compared the evolutionary histories of both filling factor and average field strength, for regions of high $\left(70^{\circ}-80^{\circ}\right)$ and low $\left(60^{\circ}-70^{\circ}\right)$ latitudes. For the south pole, we found no significant evidence of difference in the time of reversal. However, the low-latitude region of the north pole did reverse polarity much earlier than the high-latitude region. It later showed an oscillatory behavior. We suggest this may be caused by the poleward migration of flux from a large active region in 1989 with highly imbalanced flux.
\end{abstract}

\section{Introduction}

Although it is well known that most of the magnetic flux on the sun is confined to small magnetic elements with high magnetic field strength, there are few observations measuring their properties in the solar polar region. This is largely due to the difficulties associated with observing magnetic elements at an oblique angle with a longitudinal field magnetograph. This is also why most of the studies of the polar field integrate the total flux from the polar regions. Babcock (1959) first detected the reversal of the polar field by studying the evolution of the integrated polar flux. Subsequent studies of the solar magnetic cycle all used the integrated polar flux as the prime indicator of the solar field reversal (Stenflo, 1970; Howard and LaBonte, 1981; Hoeksema, 1993).

The high-resolution magnetograms of Big Bear Solar Observatory (BBSO) allowed us to study the polar field from a different perspective. The video magnetograph (VMG) system of BBSO has been observing the polar region since 1986. The data between 1986 and 1990 were studied by Tang and Wang (1991). That dataset was collected in a somewhat sporadic manner. The observations afterward 
were performed on a more regular basis. Figure 1 shows composite magnetograms of the north and south pole taken on June 12,1993. They covered regions poleward of latitude $40^{\circ}$. It shows that the polar regions, like the quiet region at lower latitude, are filled with small magnetic elements with high magnetic field. We know that the sign of the integrated polar flux reverses with a 22 -year cycle; however, we do not know how the magnetic elements evolve during the course of the solar cycle. Sheeley (1964) measured the variation of the number of polar faculae over 3 solar cycles, and found an anticorrelation with the sunspot number variation. Although faculae are known to be associated with photospheric magnetic elements and can be used to infer some of the properties of the polar region magnetic elements, they don't provide information about the sign of the magnetic elements. With the highresolution polar magnetograms, we can directly measure the various properties of the polar region magnetic elements and their evolution with the solar cycle - Does the field strength of the flux tubes change with solar cycle? Does the number of magnetic elements vary with the cycle also? Is there a difference in the evolutionary history of the positive and negative magnetic elements? These are questions we will address in this paper.

\section{The Data Set}

The data used for this study were obtained between January 1991 and June 1993. The sunspot number of solar cycle 22 reached maximum in early 1989 and did not start to decrease until late 1992 (Solar Geophysical Data, 1993). Our data cover the maximum and the declining phase of solar cycle 22. Polar field observations were performed on a regular basis with a period of about 10 days. The BBSO magnetograms are $512 \times 384$ pixels in size. The spatial resolution varies slightly, but was in general about $0.6^{\prime \prime}$ pixel $^{-1}$. The solar image size is about 3200 pixels in diameter with this resolution.

Magnetograph measurement of the polar magnetic fields at the polar regions is difficult because solar magnetic field emerges normal to the solar surface, and the magnetograph is only sensitive to the line-of-sight component of the magnetic field. The BBSO VMG system operates at video rate, taking 30 circular-polarization measurements each second. Thus there are 15 Stokes $V$ signal measurements per second. When observing sunspots and active regions, we typically integrate 256 Stokes V signal measurements for each magnetogram. To increase the signal-tonoise ratio of the polar field measurements, we integrated 4096 Stokes $V$ measurements for each polar magnetogram.

Each polar observation consists of 3 magnetograms taken at each pole, and 1 magnetogram shifted $100^{\prime \prime}$ from the polar frame for image scale calibration. Magnetic field calibration data were taken occasionally. The calibration procedure was described by Varsik (1994). Intensity images of each magnetogram were recorded simultaneously for image positioning purposes. With $0.6^{\prime \prime}$ pixel $^{-1}$ resolution, each polar magnetogram covers the region approximately $55^{\circ}$ poleward, depending on 


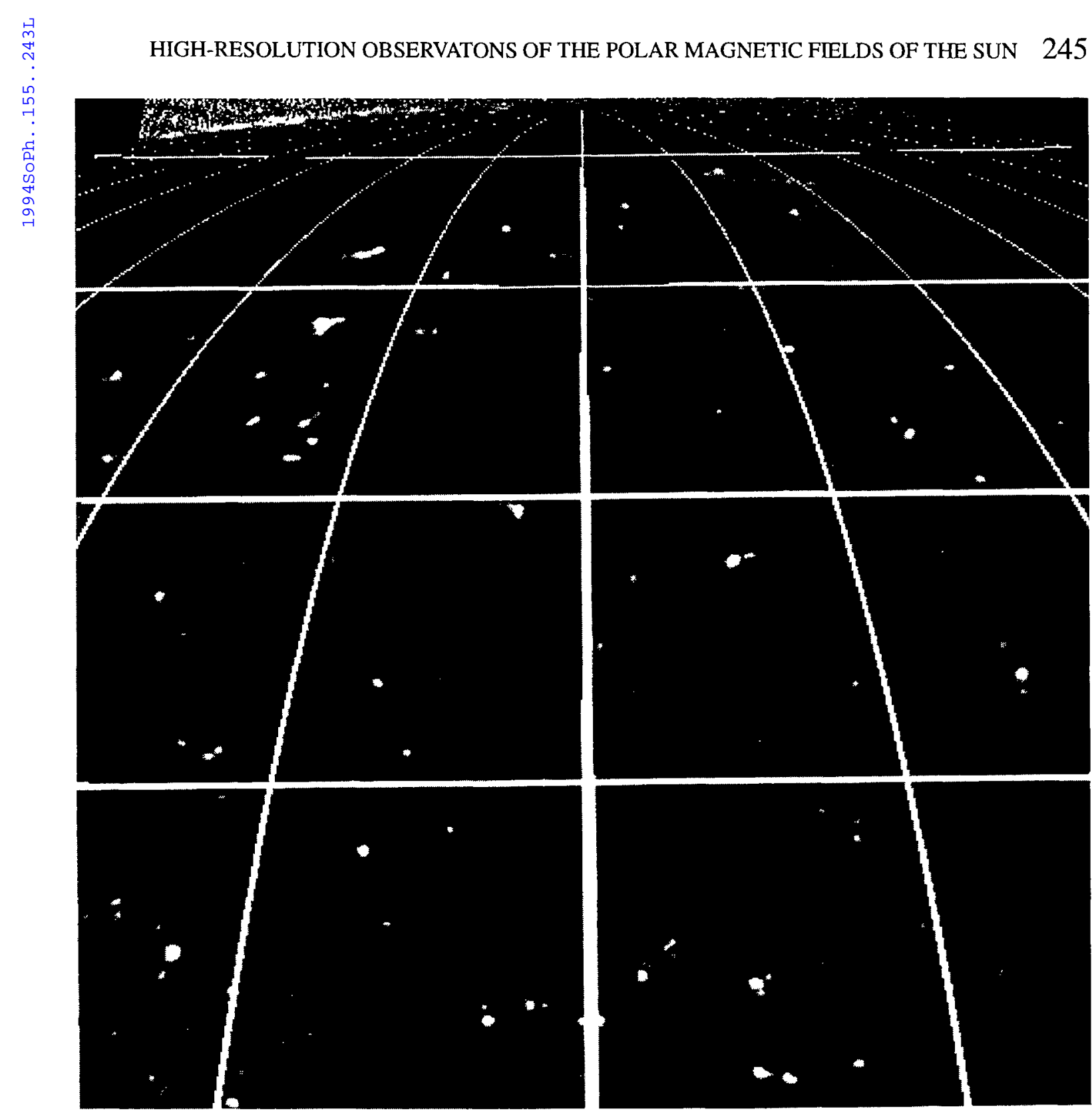

Fig. 1a.

Fig. 1a-b. Magnetograms of the north polar region (a) and south polar region (b) of June 12, 1993 with latitude and longitude lines superimposed. Each line indicates a $10^{\circ}$ increase in longitude and latitude.

the $B$ angle. The polar caps (area within $10^{\circ}$ of the poles) were only visible half of the time during the year, also due to the variation of B angle. Thus, we divided the polar magnetograms into two latitude bands, the first band between $60^{\circ}$ to $70^{\circ}$, and the second between $70^{\circ}$ and $80^{\circ}$. We will refer to these two bands as the lowand high-latitude bands hereafter. We will discuss the evolutionary history of the magnetic elements in these two latitude bands in the next section. 


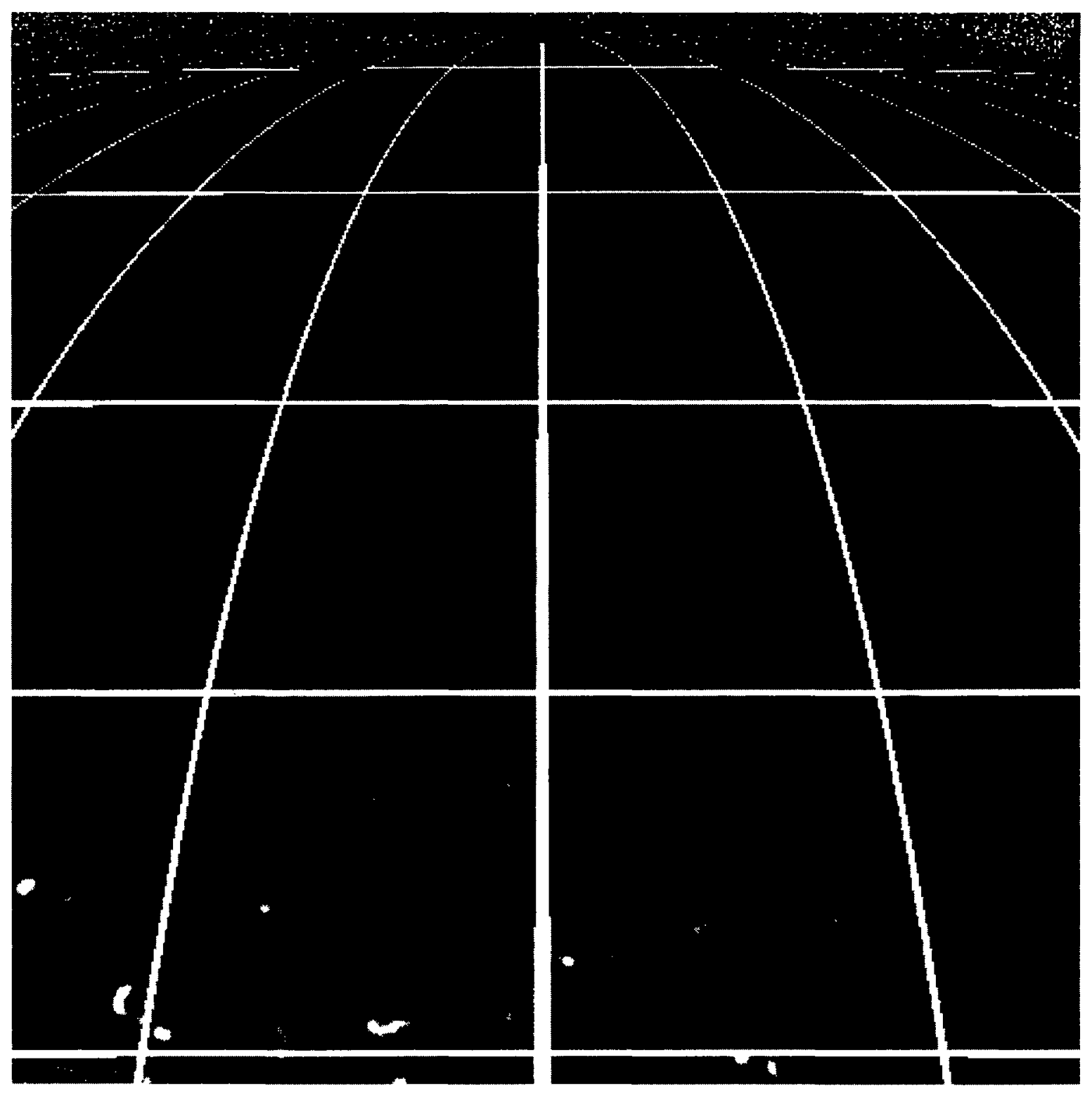

Fig. 1b.

\section{Analysis and Results}

\subsection{THE HISTOGRAM OF THE POLAR MAGNETIC FIELD}

To study the magnetic flux distribution of the polar region, we constructed the histograms $n(B)$ of magnetograms from different phases of the solar cycle as shown in the left-hand side panels of Figure 2. The maximum phase of solar cycle 22 occurred approximately around mid-1989 to late-1991 (Solar Geophysical Data, 1993), so the 1991 data represent the magnetic flux distribution during the maximum phase, and the 1993 data represent the declining phase of the cycle. First we note that they all have a symmetric central component which can be approximated by a gaussian function of the form 


$$
g(B)=A \exp \left(-\frac{\left(B-B_{0}\right)^{2}}{2 \sigma^{2}}\right) .
$$

The fitted curves were over-plotted in the left-hand side panels of Figure 2, and the differences between the measured and fitted curves, $\Delta n(B)=n(B)-g(B)$, were plotted in the right-hand side panels of Figure 2 . Note that the high-flux tails of the histograms show significant deviation from the fitted gaussian function. Examination of the magnetograms shows that the May 12, 1991 data have about equal number of positive and negative magnetic elements in the polar region, corresponding to the excess power - the excess number per bin of the histogram with respect to the fitted gaussian distribution - in both positive and negative highflux regions of the histogram. The 1993 data has predominantly positive (negative) magnetic elements at the north (south) pole, which corresponds to the excess power in the positive (negative) high-flux region of the histograms. Thus, we can describe the histograms by a two-component model - a symmetric central component $g(B)$ and a high-flux component $\Delta n(B)$. The high-flux component is clearly due to the presence of the isolated magnetic elements; however, the origin of the symmetric central component is not as obvious. Does it represent a weak background field on the solar surface? Or is it just the noise of the instrument?

To understand the origin of the symmetric central component, we note that the VMG signal $\mathrm{S}$ is the difference between the positive and negative circular polarization measurements normalized by the average of the two. That is,

$$
S=\frac{\left(I_{+}-I_{-}\right)}{\left(I_{+}+I_{-}\right)}=\frac{\Delta I}{\bar{I}} .
$$

Denote the noise in each intensity measurement by $\sigma_{ \pm}=\sqrt{I_{ \pm}}$. Then the noise of $\Delta I$ and $\bar{I}$ is $\sigma=\sqrt{\sigma_{+}^{2}+\sigma_{-}^{2}}=\sqrt{\bar{I}}$. We can rewrite Equation (2) as

$$
S=\frac{\Delta I+\sigma}{\bar{I}+\sigma} \approx \frac{\Delta I}{\bar{I}}+\frac{\sigma}{\bar{I}}=\frac{\Delta I}{\bar{I}}+\frac{1}{\sqrt{\bar{I}}} .
$$

The noise term $1 / \sqrt{\bar{I}}$ should be larger near the limb since the solar intensity is lower due to the limb-darkening effect.

We fitted the magnetic flux distributions from regions at latitudes $l=45^{\circ}$, $l=55^{\circ}, l=65^{\circ}$, and $l=75^{\circ}$, with a $10^{\circ}$ binning width. The average centroid position $B_{0}$ occurred at $B_{0} \approx 0$, and showed no $\mu(=\cos l)$ and solar-cycle dependence. Thus we believe the non-zero $B_{0}$ is due to instrumental error. The gaussian width $\sigma$ of the $75^{\circ}$ region was approximately $7 \mathrm{G}$. Figure 3 shows the ratio of the measured distribution width to the theoretical noise derived from the intensity images. The $\mu$-dependent gaussian width is consistent with the noise model derived from the intensity images. Furthermore, the gaussian width decreases if we increase the the number of frames co-added for each magnetogram. Thus, we concluded that there is no evidence of a weak uniform background field and the weak symmetric central component represents the noise level of the VMG system. 

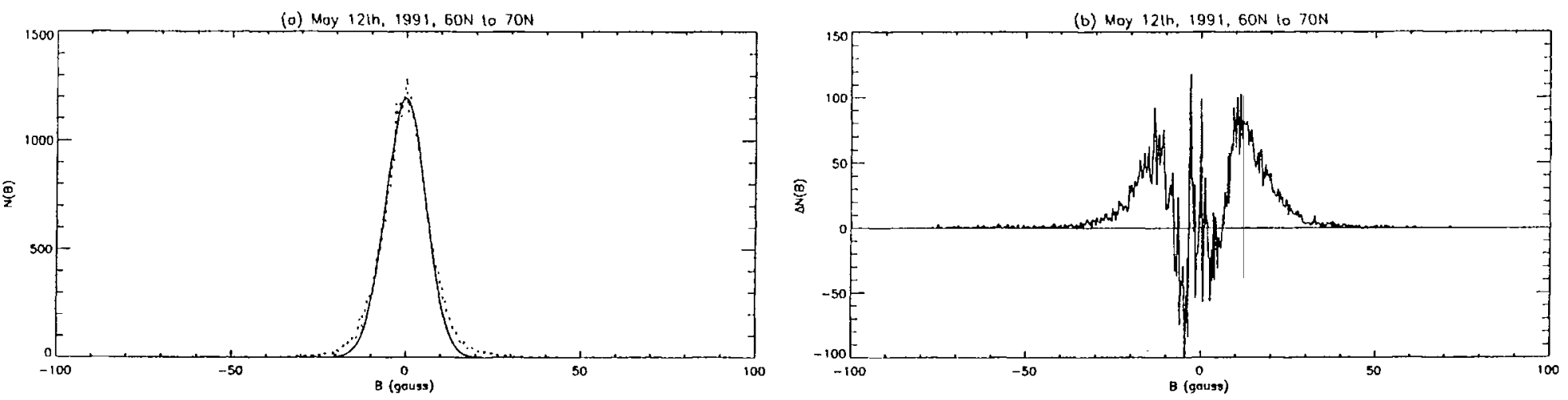

$\stackrel{N}{\infty}$
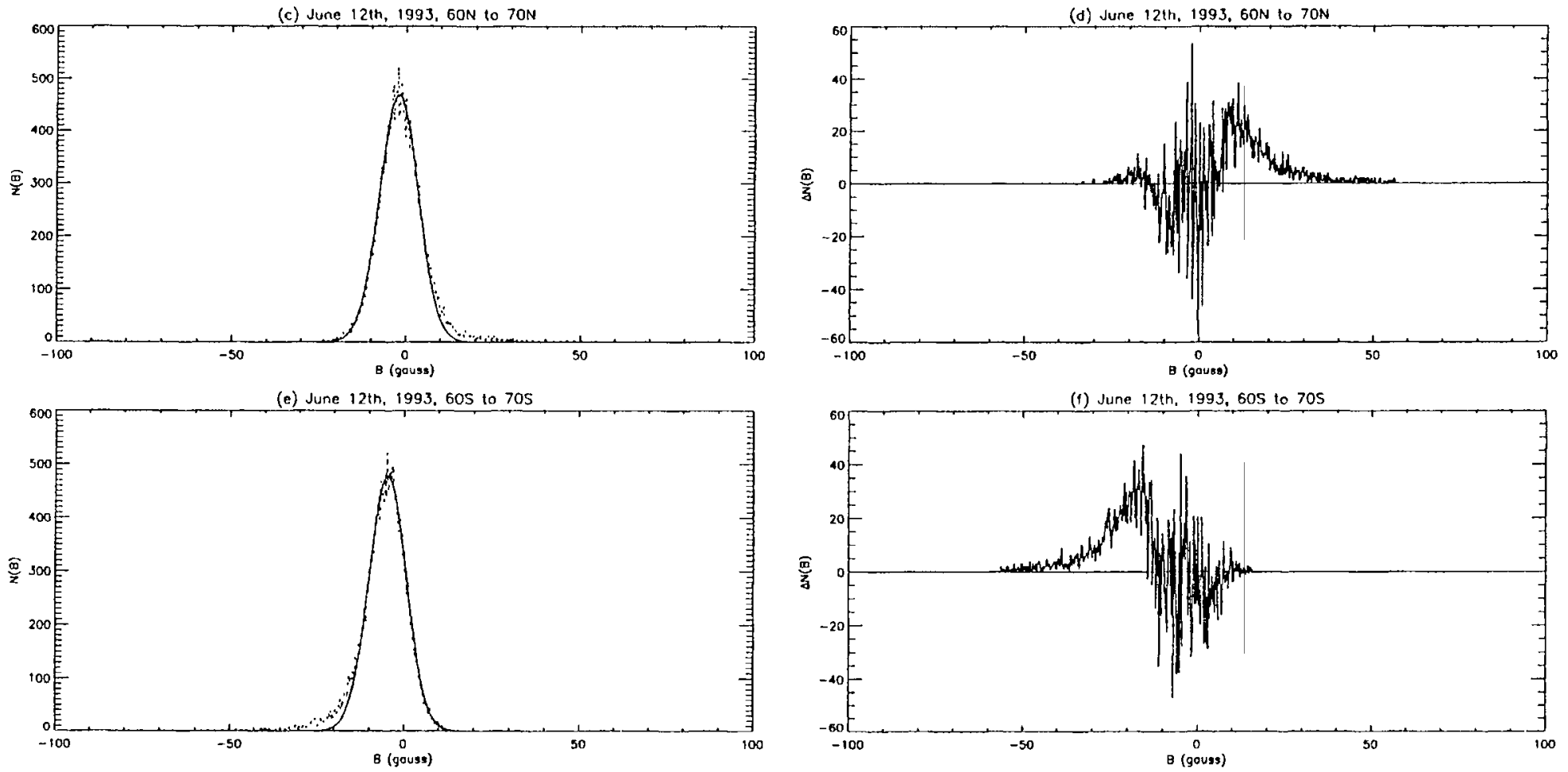

Fig. 2. Histograms of polar region magnetograms. (a), (c), and (e) flux distribution of polar region between $60^{\circ}$ and $70^{\circ}$ from May 12,1991 , June 12 , 1993 at the north pole, and June 12, 1993 at the south pole, respectively. (b), (d), and (f) flux distribution of the strong magnetic elements only from (a), (c), and (e). Dotted lines are the measured distribution, solid lines are the filtered gaussian function. 


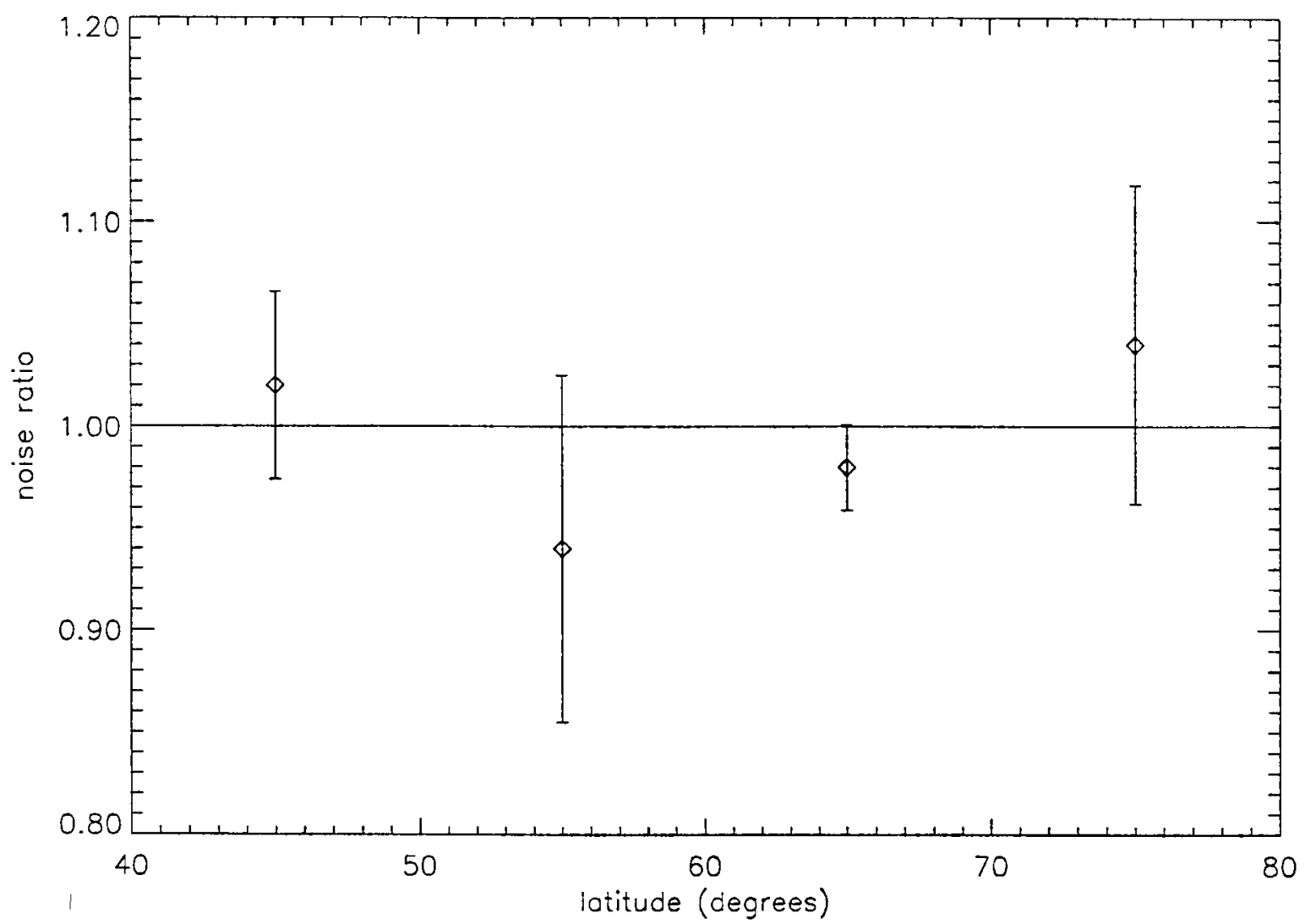

Fig. 3. The ratio of measured noise to theoretical noise derived from intensity images between latitude $45^{\circ}$ and $75^{\circ}$.

\subsection{THE INTEGRATED FLUX DENSITY}

Figure 4 shows the evolution of the integrated flux density $F$ from 1991 to 1993. The integrated flux density is defined as the total flux from a selected area averaged over the total number of pixels in the area, i.e.,

$$
F=\frac{1}{N} \sum_{B=-\infty}^{+\infty} B n(B)
$$

where $N$ is the total number of pixels within the area under consideration, $N=$ $\sum_{B=-\infty}^{+\infty} n(B)$. This is the same quantity used by other authors in the low-resolution observations.

As shown in Figure 4, the integrated flux density of the south pole reversed from positive to negative around May 1991 in both latitude bands. The high-latitude band (dotted line) was not completely reversed until early 1992. The evolution of the integrated flux density of the north pole is quite different from that of the south pole. The low-latitude band of the north pole was already reversed at the beginning of 1991. In fact, it reversed in early 1990 (Hoeksema, 1992). However, it did not increase in strength monotonically as it did in the south pole. Instead it oscillated between 0 and 1 G. It almost returned to negative polarity near the end of 1991, 


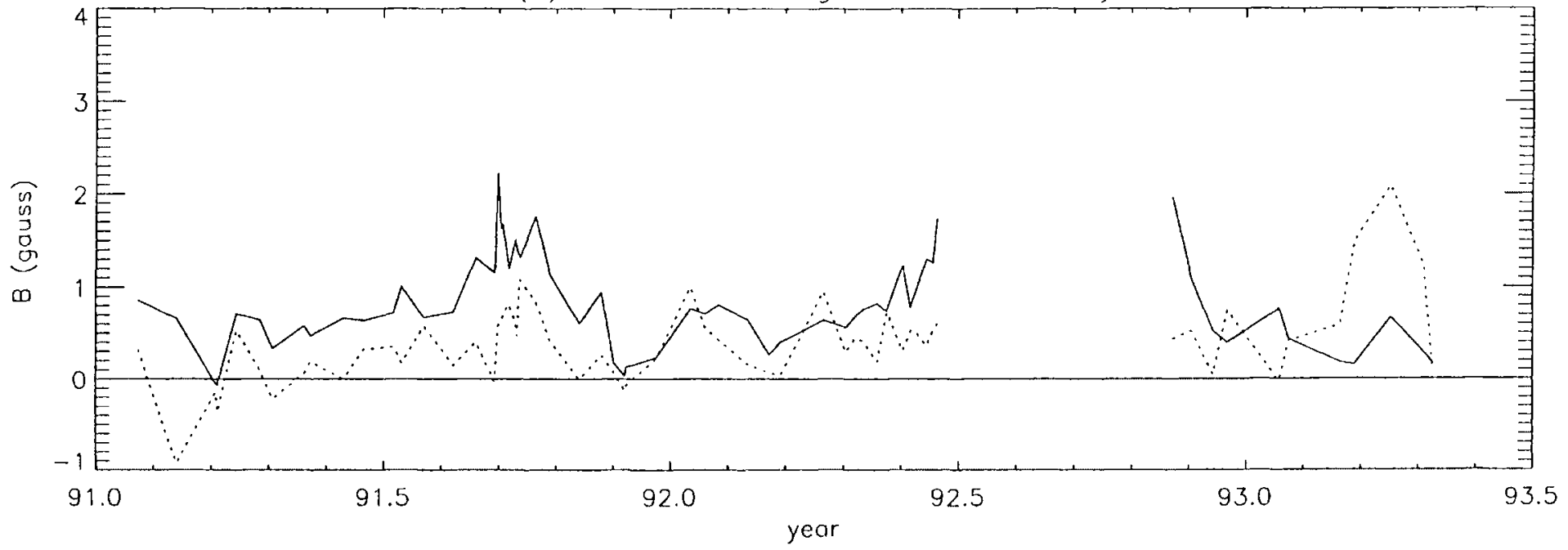

(b) South Pole Integrated Flux Density

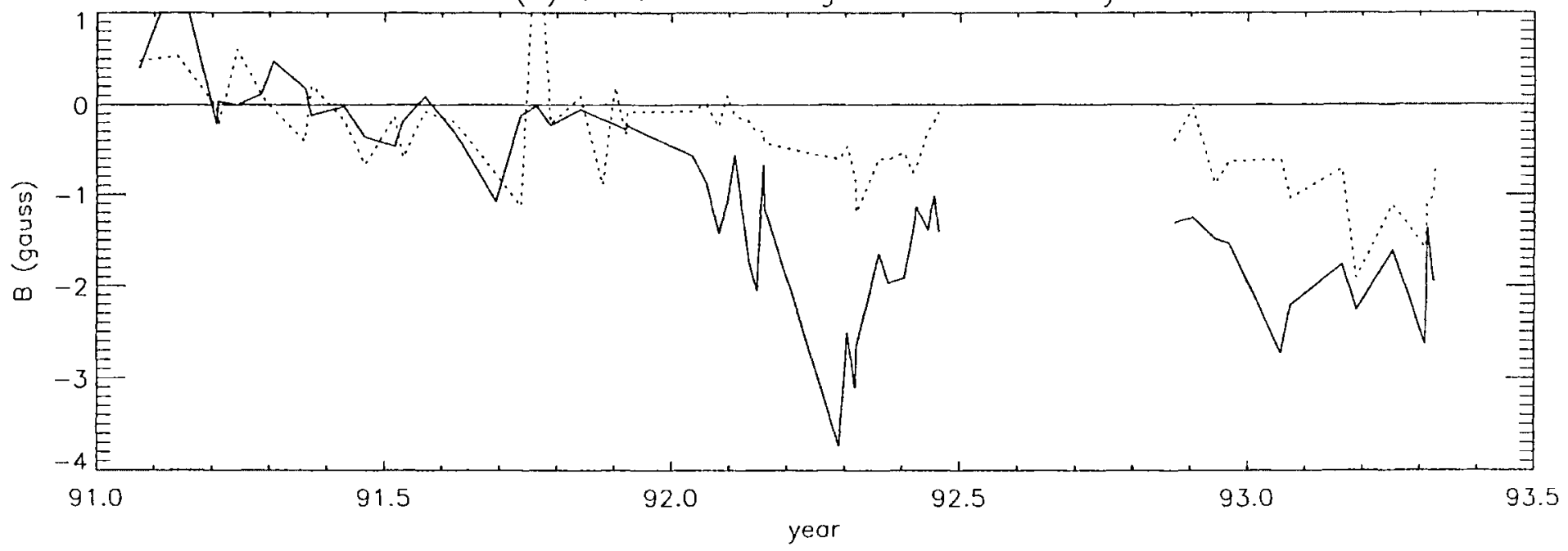

Fig. 4. The integrated flux density between latitude $60^{\circ}$ to $70^{\circ}$ (solid line), and $70^{\circ}$ to $80^{\circ}$ (dotted line) of the north pole (a), and south pole (b). There were no data between July and October 1992 due to the June 28, 1992 earthquake near Big Bear City. 
and again around April 1993. This oscillatory behavior had been observed as a common feature of the solar cycle, and was confined in the north pole after 1920 (Makarov and Sivaraman, 1989). The high-latitude band of the north pole reversed polarity at the same time as the south pole, and its evolution was basically similar to the high-latitude band of the south pole.

\subsection{THE AREA FILLING FACTOR OF THE MAGNETIC ELEMENTS}

We calculate the magnetic flux distribution $\Delta n(B)$ of the isolated magnetic elements by taking the difference between the measured histogram, $n(B)$, and the fitted gaussian function, $g(B)$, i.e., the quantity shown in the right-hand panels of Figure 2 . The area filling factors $f_{ \pm}$can then be defined as the ratio of the total number of magnetic pixels $N_{ \pm}$to the total number of pixels $N$. The + and subscripts denote the filling factor of the positive and negative magnetic elements, respectively. That is,

$$
f_{ \pm}=\frac{N_{ \pm}}{N}
$$

with $N_{ \pm}$defined by

$$
N_{ \pm}=\sum_{B=0}^{ \pm \infty} \Delta n(B) .
$$

We have to point out that the interpretation of $f_{ \pm}$as the area filling factors needs to be taken with caution. Because we are observing very close to the limb of the Sun, the projected area covered in each pixel has strong $\mu$ dependence. Thus, $N_{ \pm}$and $N$ are not accurate measurements of area. However, if the range of $\mu$ of the region under consideration is sufficiently small, the error is small and can be ignored.

The evolution of the area filling factors of the magnetic elements is plotted in Figure 5. From the evolution of the integrated flux density, polar field reversal occurred during 1991. During the same period the polar regions above $70^{\circ}$ were populated by magnetic elements with mixed polarity. The filling factors of both polarities in the high-latitude band were about equal. This period of mixed polarities lasted for an extended period of time and it was only in late 1992 that the polarity of the magnetic elements at the north pole became predominantly positive and that of the south pole became predominantly negative. In the low-latitude bands, the south-pole data show a clear sign of polarity reversal around April 1991. The positive magnetic elements filling factor decreases gradually while that of the negative magnetic elements increases. By early 1992, the low-latitude region in the south pole had become predominantly populated by negative magnetic elements. The situation at the north pole was very different. The magnetic elements in the low-latitude region of the north pole reversed to positive polarity before 1991. It is intriguing to note that while the low-latitude region in the north pole reversed 


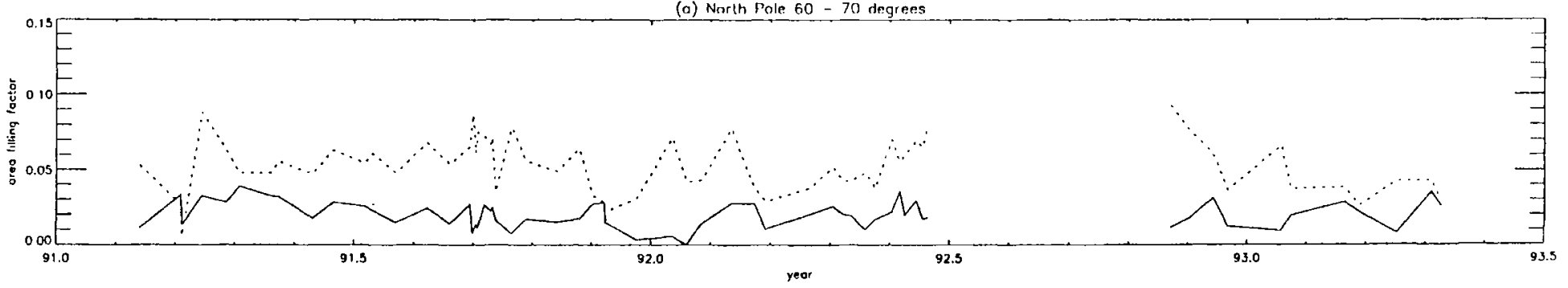

(b) North Pole $70-80$ degrees
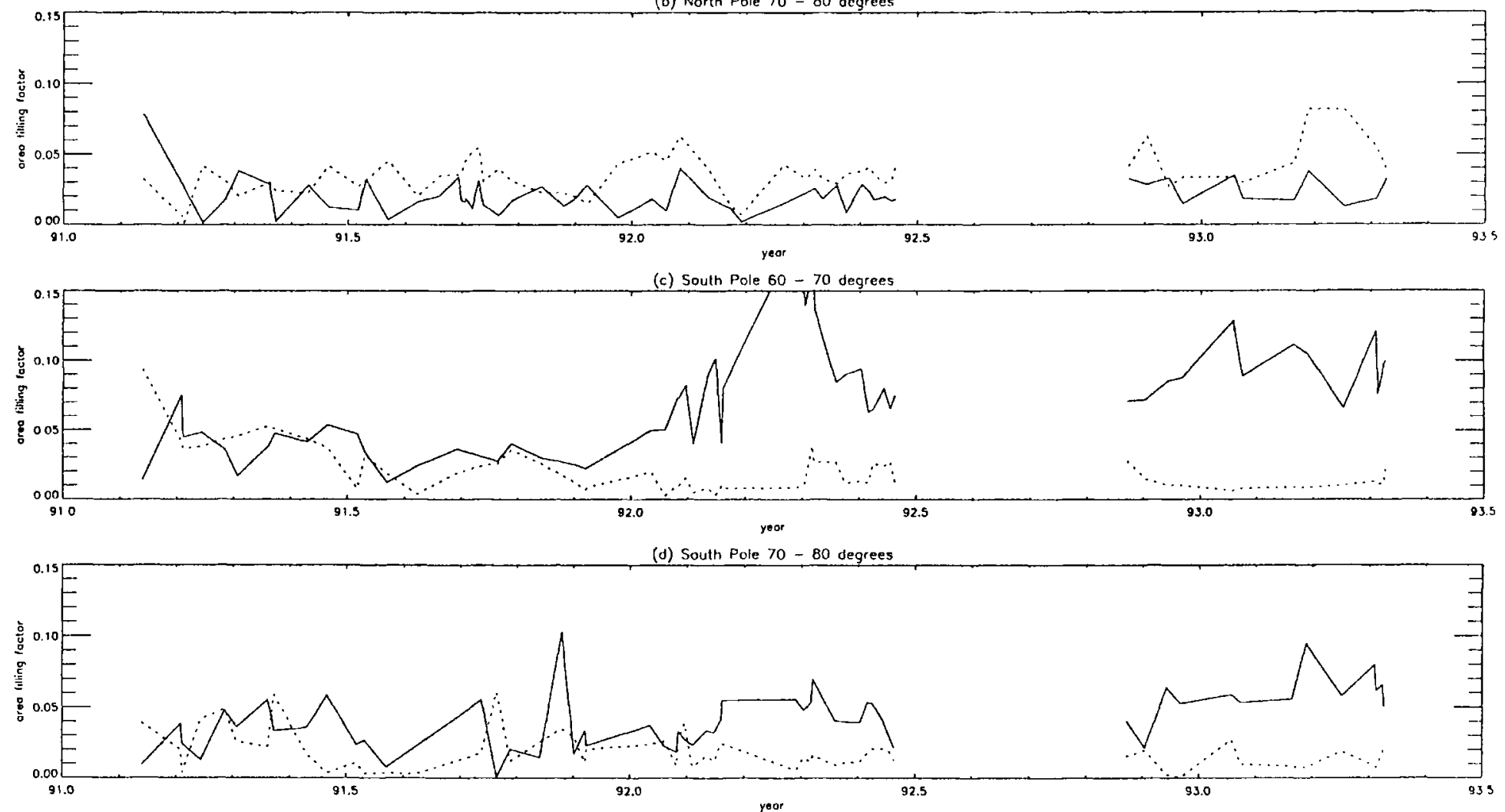

Fig. 5. The area filling factors of the magnetic elements in the polar regions. Solid lines are the filling factors of the negative magnetic elements, and dotted lines are the positive magnetic elements. 
earlier than the same latitude region at the south pole, the region above $70^{\circ}$ reversed polarity at almost the same time at both poles.

\subsection{THE AVERAGE FIELD STRENGTH OF THE MAGNETIC ELEMENTS}

We have shown that the increase in integrated polar flux density was accompanied by the increase of the filling factor for one polarity and the decrease of $f$ for the other polarity. Does the average field strength of the magnetic elements show similar evolution? The average field strength is defined as follows:

$$
\bar{B}_{ \pm}=\frac{1}{N_{ \pm 0}} B \Delta n(B) .
$$

Again the + and - subscripts denote the average field strength of the positive and negative magnetic elements, respectively. Figures 6(a) and 6(c) show the evolution of the average field strength of the positive and negative magnetic elements between 1991 and 1993. Note that the short time-scale variations of the two polarities are mostly symmetric, indicating a variation in the magnetic field calibration procedure. Nevertheless this calibration error is small most of the time, and does not prevent us from deducing useful results. During 1991, it is difficult to tell if the average field strength of the positive and negative are different. However, it is clear that starting from late 1992 the average field strength of the dominant polarity became higher than the average field strength of the other polarity. To illustrate the evolution of the average field strength more clearly, we plotted the 'polarization' $p$ of the magnetic field elements, i.e.,

$$
p=\frac{\left|\bar{B}_{+}\right|-\left|\bar{B}_{-}\right|}{\left|\bar{B}_{+}\right|+\left|\bar{B}_{-}\right|},
$$

in Figures 6(b) and 6(d). The polarization of both the low- and high-latitude bands of the north pole show oscillatory behavior between early-1991 and mid-1992 and they were 'polarized' to the positive polarity after 1993. At the south pole, both the low- and high-latitude bands were about equally polarized between 1991 and mid-1992. After 1993 both latitudes bands were strongly 'polarized' toward the negative polarity. This indicates that after 1993, the average field strength of the positive magnetic elements is higher than that of the negative magnetic elements at the north pole, and at the south pole, the negative magnetic elements have a higher field strength. Thus, both the number of magnetic elements and the average field strength of the dominant polarity increase as the polar field builds up to its maximum.

Note that the average field strengths of the low- and high-latitude bands are almost identical at both poles. One possible explanation is that the longitudinalfield magnetograph integrates the magnetic signal along the line-of-sight over the region defined by the CCD pixels. When observing near the limb, each pixel actually covers an solar area $\mu^{-1}$ times the solar area observed at disk-center. Since 

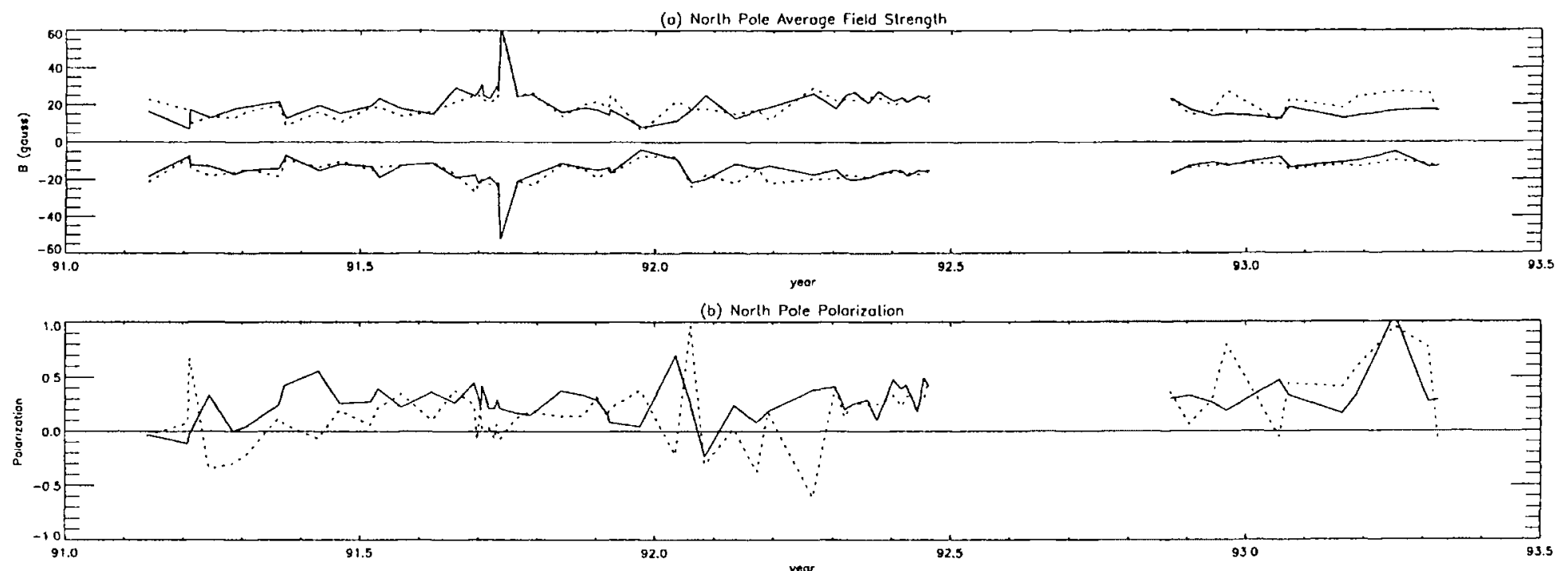

(c) South Pole Averoge field Strength
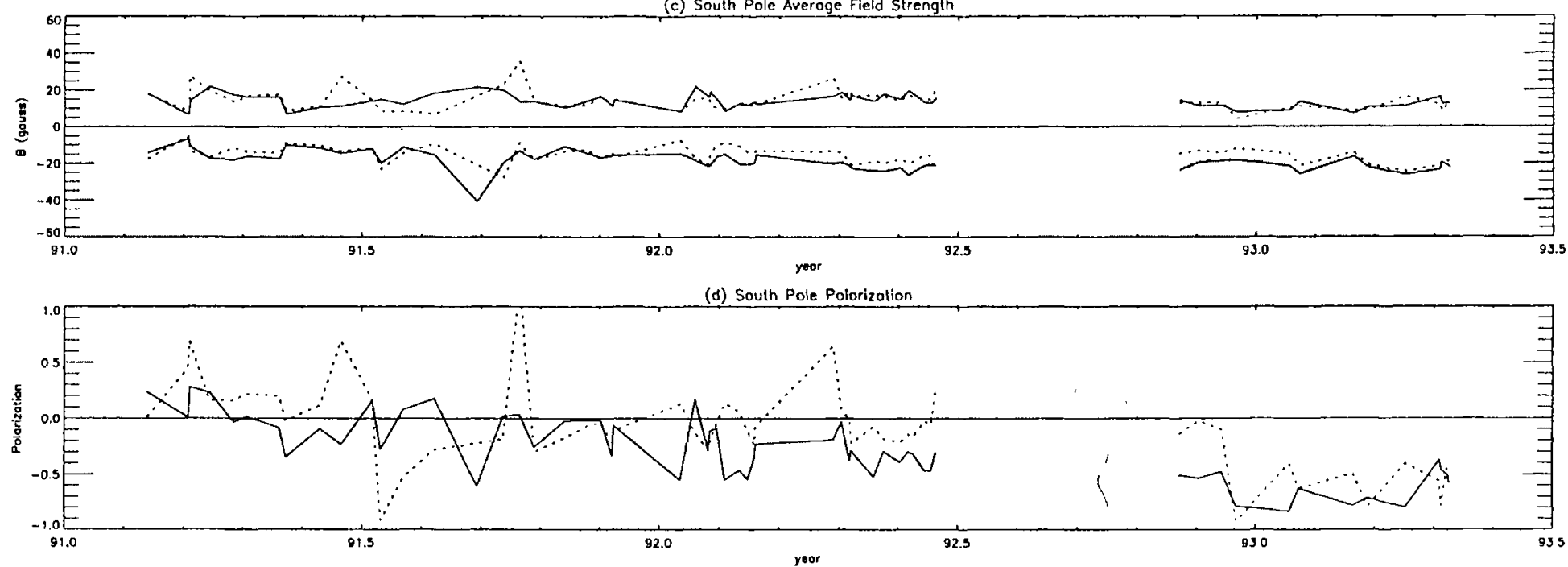

Fig. 6. The average field strength and 'polarization' (as defined in Section 3.4) of the magnetic elements in the polar regions. (a) North pole average field strength, (b) north pole polarization, (c) south pole average field strength, and (d) south pole polarization. Solid lines represent the low-latitude bands, and dotted lines are for the high-latitude bands. 
the average field strength was calculated with only contribution from the strong flux elements, we can assume a constant filling factor in these elements. If the solar magnetic field emerges with $90^{\circ}$ angle to the surface, then the line-of-sight component of the field is $B \mu$. The $\mu$ factor in the longitudinal field and the $\mu^{-1}$ factor in the area term cancel each other, resulting in an $\mu$-independent average magnetic field strength.

\section{Conclusions and Discussions}

Using the BBSO high-resolution magnetograms, we demonstrated that the magnetic flux in the polar regions is concentrated in small magnetic elements. We showed the evolution of the integrated polar flux density, the area filling factors and the average field strength of these magnetic elements during the period between January 1991 and June 1993. We can summarize the results of this study as follows:

(1) During the course of polar field reversal, the polar region magnetic elements evolve from predominantly of one polarity to mixed polarities when the integrated flux density reversal occurs, and finally become predominantly of the opposite polarity.

(2) The average field strength of the magnetic elements in the polar region has a solar-cycle dependence. The average field strength of the dominant polarity field elements at the pole increases with the rising phase of the polar field, while the average field strength of the opposite polarity field elements decreases.

(3) The integrated flux density of regions between $60^{\circ} \mathrm{S}$ to $70^{\circ} \mathrm{S}$ and $70^{\circ} \mathrm{S}$ to $80^{\circ} \mathrm{S}$ at the south pole were observed to reverse at about the same time. At the north pole, there was a phase difference of the time of reversal. The low-latitude region reversed first. The high-latitude region reversed at the same time as the south pole. The low-latitude band also showed oscillatory behavior.

It is generally agreed that the polar fields arise from the accumulation of the flux of the following polarity of decaying active regions (Leighton, 1964; Howard and LaBonte, 1981; DeVore and Sheeley, 1987). If this is the case, the poleward migration of the flux should introduce a phase lag in the time of reversal in the highlatitude regions. The low-latitude region of the north pole did reverse much earlier than the high-latitude region; however, the south pole showed almost simultaneous reversal of high-and low-latitude regions. The only indication of a phase difference of reversal time in the south pole may be that the high-latitude band did have a extended period of mixed polarity phase compared with the monotonic decrease of the integrated flux density of the low-latitude bands. So, what caused the early reversal of the north pole low-latitude region? Interestingly the large active region of 1989, NOAA AR 5395 (Wang et al., 1991), appeared at latitude $33^{\circ} \mathrm{N}$ with large apparent flux imbalance $-80 \%$ of the flux was of positive polarity. It is possible that this excess positive flux was transported poleward and caused the early reversal of the low-latitude band of the north pole. If this is indeed the case, it provides strong 
evidence for the poleward migration of flux. Nevertheless, the lack of phase lag of the reversal time of the south pole indicates otherwise and remains to be explained.

In a study of non-radial magnetic field in the photosphere, Pope and Mosher (1975) suggested that the polar magnetic field exists in the form of bipolar pairs with N-S alignments and widely differing orientation angles so that the integrated flux shows a net field. Murray (1992) studied the photospheric magnetic field distribution and the $\mu$ dependence of the visibility of field elements. He concluded that isolated weak magnetic elements are highly inclined with respect to the normal vector of the solar surface, and the inclination angle of magnetic elements with magnetic strength between 100 to $300 \mathrm{G}$ is about $30^{\circ}$. For magnetic elements with field strength below $100 \mathrm{G}$, the inclination angle is even larger, about $48^{\circ}$. From our observations, the majority of magnetic elements at the south pole were of negative polarity and there were few magnetic elements of positive polarity $20^{\circ}$ within the pole by early 1993 . If the inclination angles of the flux tubes are as large as Murray suggested, we should see a substantial number of positive magnetic elements with strength between 20 to $50 \mathrm{G}$ near latitude $70^{\circ}$. Our high-resolution polar magnetograms clearly showed that is not the case. The identical average field strength of high- and low-latitude regions derived in Section 3.6 also implies that the magnetic elements emerge normal to the solar surface.

\section{Acknowledgements}

We would like to thank the observers at Big Bear Solar Observatory for their efforts in collecting the data. HL is grateful to $\mathrm{Dr} \mathrm{H}$. Wang for many discussions. This research is supported by ONR grant number N00014-89-J-1069. The Big Bear Solar Observatory is supported by NASA grant number NAGW-1972, and NSF grant number ATM-9122023.

\section{References}

Babcock, H.D.: 1959, Astrophys. J. 130, 364.

DeVore, R. C. and Sheeley, N. R.: 1987, Solar Phys. 108, 47.

Hoeksema, T. J.: 1993, Geomagnetism and Geoelectricity, preprint.

Howard, R. and LaBonte, B. J.: 1981, Solar Phys. 74, 131.

Leighton, R. B.: 1964, Astrophys. J. 140, 1547.

Makarov, V. I. and Sivaraman, K. R.: 1989, Solar Phys. 123, 367.

Murray, N.: 1992, Astrophys. J. 401, 386.

Pope, T. and Mosher, J.: 1975, Solar Phys. 44, 3.

Sheeley, N. R.: 1964, Astrophys. J. 140, 731.

Stenflo, J, O.: 1970, Solar Phys. 13, 42.

Tang, F. and Wang, H.: 1991, Solar Phys. 132, 247.

Varsik, J.: 1994, Solar Phys., submitted.

Wang, H., Tang, F., Zirin, H., and Ai, G.: 1991, Astrophys. J. 380, 282. 